\title{
PERIODICALLY PERTURBED CONSERVATIVE SYSTEMS
}

\author{
BY SHAIR AHMAD
}

Communicated by Francois Treves, June 28, 1973

In this note we announce a result concerning the existence of a periodic solution for a class of periodically perturbed conservative systems. Our result, in a sense, completes a series of investigations originated by W. S. Loud [4]. Also see [1], [2], [3], and [5]. Our techniques are different from those of the authors cited above.

Consider the vector differential equation

$$
x^{\prime \prime}+\operatorname{grad} G(x)=p(t)=p(t+2 \pi),
$$

where $p \in C\left(R, R^{n}\right), G \in C^{2}\left(R^{n}, R\right)$. This equation can be interpreted as the newtonian equation of a mechanical system subject to conservative internal forces and periodical external forces.

THEOREM 1 (LAZER [1]). Let $A$ and $B$ be real constant symmetric matrices such that if $\lambda_{1} \leqq \lambda_{2} \leqq \cdots \leqq \lambda_{n}$ and $\mu_{1} \leqq \mu_{2} \leqq \cdots \leqq \mu_{n}$ denote the eigenvalues of $A$ and $B$ respectively then there exist integers $N_{k} \geqq 0, k=$ $1, \cdots, n$, such that

$$
N_{k}^{2}<\lambda_{k} \leqq \mu_{k}<\left(N_{k}+1\right)^{2} .
$$

If, for all $a \in R^{n}, A \leqq \partial^{2} G(a) / \partial x_{i} \partial x_{j} \leqq B$, then (1) has at most one $2 \pi$ periodic solution.

Our theorem establishes the existence part of the preceding theorem. More specifically, we prove

Theorem (1)*. If $G, A$ and $B$ satisfy the hypothesis of Theorem 1, then (1) has a $2 \pi$-periodic solution.

The key to the proof of our theorem is

LEMMA 1. Let $\bar{Q}(t)$ be a real $n \times n$ symmetric matrix whose elements are bounded, measurable and $2 \pi$-periodic on the real line. Let $A$ and $B$ be real constant symmetric matrices such that $A \leqq Q(t) \leqq B$. If $\lambda_{1} \leqq \cdots \leqq \lambda_{n}$ and $\mu_{1} \leqq \cdots \leqq \mu_{n}$ denote the eigenvalues of $A$ and $B$ respectively then there

AMS (MOS) subject classifications (1970). Primary 34C25, 34A10. 
exist integers $N_{k} \geqq 0, k=1, \cdots, n$, satisfying

$$
N_{k}^{2}<\lambda_{k} \leqq \mu_{k}<\left(N_{k}+1\right)^{2} .
$$

Let $f(t)$ be a real vector-valued $2 \pi$-periodic continuous function with $\|f(t)\| \leqq K, K$ some number. Then there exists a number $r>0$, independent of $f(t)$, such that for any periodic solution $u$ of $u^{\prime \prime}+\bar{Q} u=f$ the inequality $\|u(t)\|^{2}+\left\|u^{\prime}(t)\right\|^{2} \leqq r^{2}$ holds for all $t$ (we mean $u^{\prime}$ absolutely continuous and the preceding equation holds a.e.).

Using this lemma we prove that our theorem follows from a generalization of Poincaré's perturbation theorem (see [3]). The proof of Lemma 1 is too long to give here. A brief sketch may be given along the following line. Assuming that the conclusion of Lemma 1 is false, we construct a sequence of equations of the form

$$
z_{m}^{\prime \prime}+Q_{m}(t) z_{m}=g_{m}(t) \text { a.e. }
$$

where $z_{m}, Q_{m}$ and $g_{m}$ are $2 \pi$-periodic ( $Q_{m}$ symmetric). It is shown that the sequences $\left\{z_{m}\right\}$ and $\left\{z_{m}^{\prime}\right\}$ are uniformly bounded and equicontinuous, and $\left\{Q_{m}\right\}$ weakly converges to some matrix $Q(t)$. Using the fact that the set of symmetric $n \times n$ matrices $S$ satisfying $A \leqq S \leqq B$ can be considered as a compact convex subset of $R^{p}, p=n(n+1) / 2$, it follows from Lemma $1 \mathrm{~A}$ of (p. 157 of [5]) that $Q(t)$ is a $2 \pi$-periodic symmetric matrix and $A \leqq Q(t) \leqq B$. It is then shown that this leads to a contradiction of Theorem 1 of [1].

\section{REFERENCES}

1. A. C. Lazer, Application of a lemma on bilinear forms to a problem in nonlinear oscillations, Proc. Amer. Math. Soc. 33 (1972), 89-94. MR 45 \#2258.

2. A. C. Lazer and D. A. Sánchez, On periodically perturbed conservative systems, Michigan Math. J. 16 (1969), 193-200. MR 39 \#7212.

3. D. E. Leach, On Poincarés perturbation theorem and a theorem of W. S. Loud, J. Differential Equations 7 (1970), 34-53. MR 40 \#4539.

4. W. S. Loud, Periodic solutions of nonlinear differential equations of Duffing type, Proc. United States-Japan Seminar on Differential and Functional Equations (Minneapolis, Minn., 1967), Benjamin, New York, 1967, pp. 199-224. MR 36 \#6704.

5. E. B. Lee and L. Markus, Foundations of optimal control theory, Wiley, New York, 1967. MR 36 \#3596.

Department of Mathematics, Oklahoma State University, Stillwater, OKLAHOMA 74074 